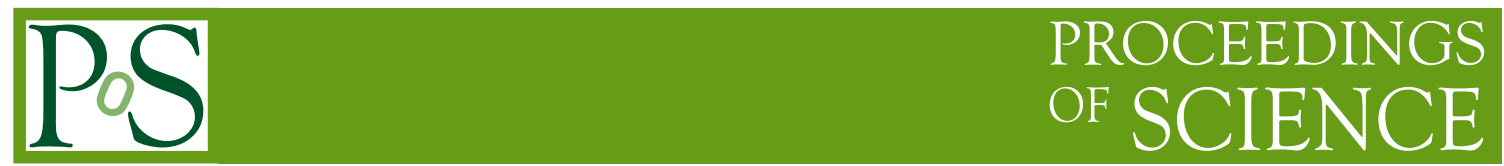

\title{
Lattice QCD at Finite Density and Temperature
}

\author{
Harvey B. Meyer* \\ Johannes Gutenberg-Universität Mainz \\ Institut für Kernphysik \\ D-55099 Mainz, Germany \\ E-mail: meyerh@kph.uni-mainz.de
}

I review the latest calculations in finite temperature QCD on the lattice, including the chiral aspects of the phase transition, quark number susceptibilities and their comparison with the hadron resonance gas model. I also attempt to summarize the state of our knowledge of the QCD phase diagram at small to moderate baryon chemical potential.

Sixth International Conference on Quarks and Nuclear Physics,

April 16-20, 2012

Ecole Polytechnique, Palaiseau, Paris

*Speaker. 


\section{Introduction}

The study of the QCD phase diagram started even before the discovery of asymptotic freedom. One early question was the high-temperature fate of a gas of hadrons with an ever increasing density of states. After the advent of QCD, important developments in our understanding of thermal field theory took place. The motivation was, and to a large extent still is, early universe cosmology, since for a time on the order of the microsecond the temperature was high enough for the elementary degrees of freedom of QCD to be in a deconfined state. Experimentally, the access to such extreme conditions of temperature is possible through heavy-ion collisions (see for instance the overview given by J.-P. Blaizot in these proceedings). Since an extremely dynamical system is created in these collisions, making contact with the equilibrium properties of finite-temperature QCD is a non-trivial task which motivates in turn that a robust control over these properties be reached computationally. The leading ab-initio computational method to achieve this goal is the MonteCarlo importance sampling of the lattice-regularized QCD path integral. An educated guess for the equation of state could be made by the early eighties (see for instance [1]), but it was impossible to infer the order of the QCD transition (1st order, 2nd order or crossover) based on general arguments, and showing convincingly that it is a crossover is a unique achievement of lattice QCD [2, 3, 4].

In this review for non-experts we consider the phase diagram as a function of temperature and baryon chemical potential (see [5] for an introduction to the subject). A representation of our current knowledge of the QCD phase diagram from lattice simulations is displayed in Fig. 1 . First, at vanishing chemical potential the transition from the low-temperature, hadronic phase to the high-temperature, deconfined and chirally symmetric phase, is a crossover [2, 3, 切. Second, the transition temperature $T_{c}$ is only weakly dependent on the chemical potential [6, 7]. As a rule of thumb, to lower $T_{c}$ by $10 \mathrm{MeV}$, the chemical potential must be turned up to half the nucleon mass. Furthermore, it has been shown that the width of the transition is initially only weakly dependent on the chemical potential [6] (see Sec. B). What happens beyond $\mu_{B}=500 \mathrm{MeV}$ remains, however, uncertain, due to the limitation of the current Monte-Carlo methods.

\section{Static quantities at $\mu_{B}=0$}

The thermodynamic properties of QCD have been calculated with different lattice actions. The most commonly used action, with so far the most complete results, is the rooted staggered formulation of lattice QCD. Even within this formulation, a lot of freedom remains to completely specify the action. The most recently used actions are the 'stout' action [8] and the 'HISQ' action [9]. Typical criteria used to optimize the choice of action are the requirement of a continuum-like dispersion relation and small mass splittings among the sixteen pseudoscalar bosons. Indeed, in the hadronic phase the latter turn out to be the dominant source of discretization errors on the static quantities. At this point, good agreement has been reached between the Budapest-Wuppertal and the hotQCD collaborations, in particular for the renormalized Polyakov loop and the quark number susceptibilities [8, 9].

The WHOT collaboration obtained results with $(\mathrm{O}(a)$ improved) Wilson fermions using the socalled fixed-scale approach, which means that the temperature is varied by changing $N_{t}[10]$. This has the significant advantage that the bare parameters of the theory do not need to be tuned to yield 


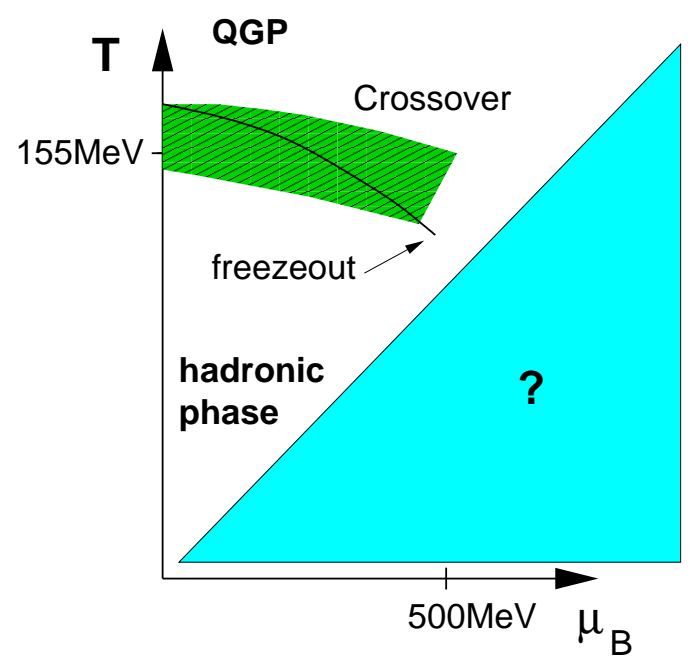

Figure 1: Status of our knowledge of the phase diagram at small $\mu_{B}$ from lattice QCD. For comparison the freezeout curve determined in heavy ion collisions is sketched as well.

a 'line of constant physics' where the renormalized quark masses are kept fixed in physical units. In this approach the discretization errors behave radically differently: they become large at high temperatures, where $N_{t}$ is no longer large enough. This also makes the approach complementary to the fixed- $N_{t}$ method that has been pursued with the staggered formulation. The results obtained so far are not competitive yet from the point of view of statistical accuracy, but it should be pointed out that in these calculations $N_{t} \geq 12$ in the hadronic phase.

Although computationally more expensive, domain wall fermions have the advantage over Wilson fermions that the lines of constant physics are easier to determine and they facilitate the computation of certain chiral observables. See [11] for a recent calculation.

Since the QCD transition is a crossover, different definitions of the transition temperature can lead to different results. The Budapest-Wuppertal collaboration obtains, from the peak of the chiral susceptibility $\chi_{\bar{\psi} \psi} / T^{4}, T_{c}=147(2)(3) \mathrm{MeV}$, and from the inflection point of $m_{\mathrm{q}}\langle\bar{\psi} \psi\rangle / m_{\pi}^{4}$, $T_{c}=155(3)(3) \mathrm{MeV}[3]$. The hotQCD collaboration proceeds by performing an $\mathrm{O}(4)$ scaling fit to the chiral condensate and chiral susceptibility and obtains $T_{c}=(154 \pm 9) \mathrm{MeV}$ [ $₫$ ].

Let

$$
\frac{f(T, \vec{\mu})}{T^{4}}=-\frac{1}{V T^{3}} \log Z(V, T, \vec{\mu}), \quad \vec{\mu} \equiv\left(\mu_{B}, \mu_{S}, \mu_{Q}\right)
$$

be the free energy in the presence of baryon, strangeness and electric charge chemical potentials. Recently, the quark number susceptibilities, for instance

$$
\chi_{2}^{S} \equiv-\frac{\partial^{2} f}{\partial \mu_{S}^{2}}
$$

for strangeness, have been investigated in detail with an eye on systematic effects. These observables are expected to change substantially when the parton degrees of freedom deconfine, because at sufficiently high temperature the quark flavor quantum number is carried by quark quasiparticles rather than by hadronic quasiparticles. Fig. (2) displays the three susceptibilities obtained by two independent lattice collaborations [8, Q] Their results are in good agreement and they provide good 

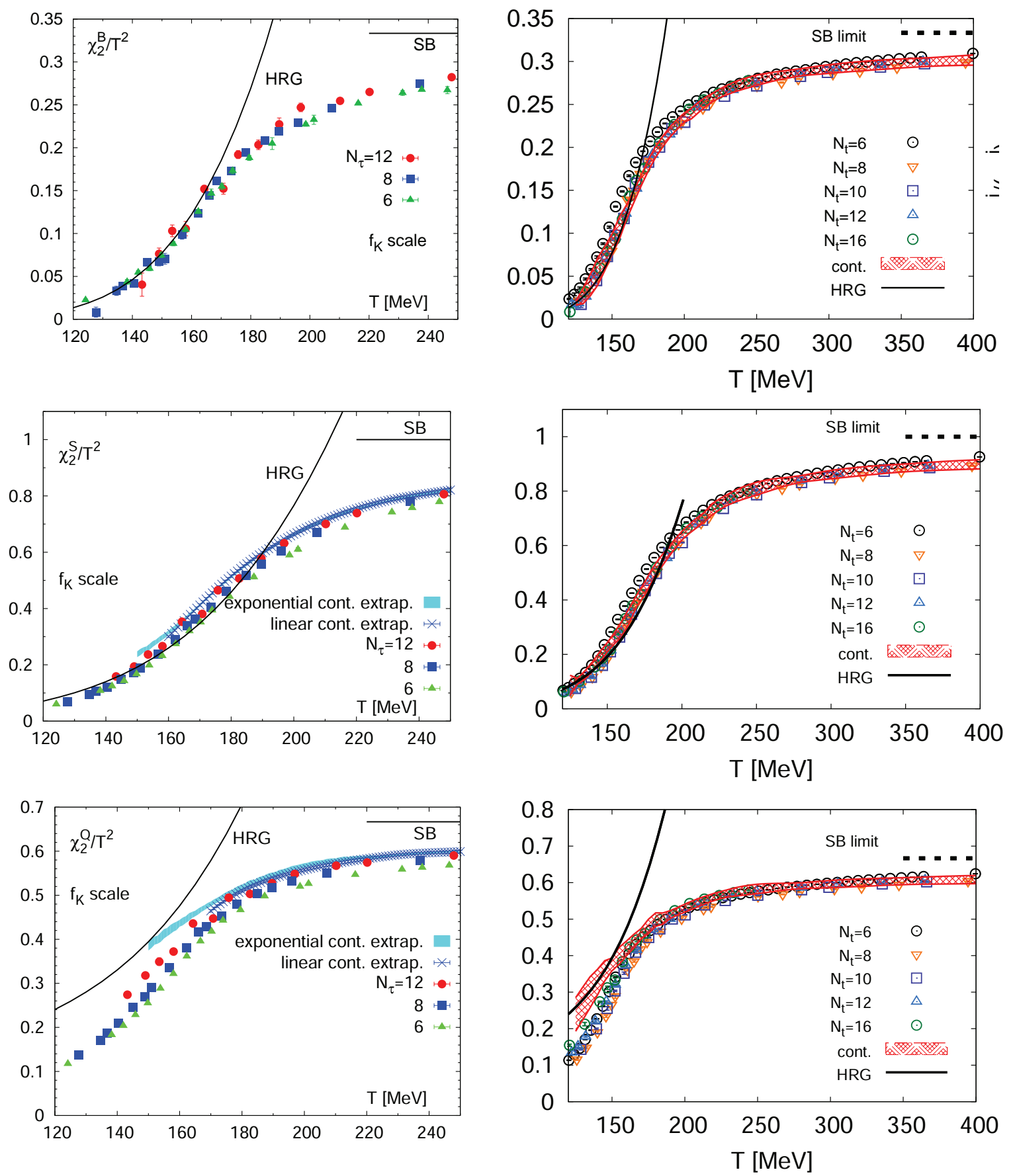

Figure 2: Top: baryon number susceptibility. Middle: strangeness susceptibility. Bottom: electric charge susceptibility. The left plots are taken from [9] (hotQCD collaboration) and the right plots from [8] (Budapest-Wuppertal collaboration). 
evidence that discretization errors are under control in the crossover region. The baryon number susceptibility is at the same time the first non-trivial Taylor coefficient in the expansion of the free energy in the baryon chemical potential (see next section).

The electric charge fluctuations have a relatively precocious inflection point around $155 \mathrm{MeV}$. One notices that the inflection point of the strangeness susceptibility is located about $15 \mathrm{MeV}$ above that value. A recent calculation of the charm fluctuations presented by C. Ratti at this conference indicates that the charm susceptibility rises much later. Finally, the expectation value of the renormalized Polyakov loop, which measures the free energy of a static quark, has an inflection point around $200 \mathrm{MeV}$ [4]. Thus it appears that the heavier the carrier of the fundamental color charge, the later its properties go over to their high-temperature behavior.

\subsection{Comparison with the hadron resonance gas model (HRG)}

Consider an ideal gas of hadrons with the free energy $f(T, \vec{\mu})=\sum_{i \in \text { species }} f_{i}(T, \vec{\mu})$,

$$
\frac{f_{i}(T, \vec{\mu})}{T^{4}}=-\frac{d_{i}}{2 \pi^{2}} \frac{M_{i}^{2}}{T^{2}} \sum_{k=1}^{\infty}( \pm)^{k+1} \frac{z_{i}^{k}}{k^{2}} K_{2}\left(k M_{i} / T\right), \quad z_{i}=\exp \left[\left(B_{i} \mu_{B}+Q_{i} \mu_{Q}+S_{i} \mu_{S}\right) / T\right]
$$

The upper sign corresponds to mesons and the lower sign to baryons and $d_{i}$ is the multiplicity factor. Although most hadrons are unstable, it has been shown a long time ago [12, 13] that if hadron interactions proceed dominantly through resonant scattering, it is appropriate to sum over those states that have a width $\Gamma \lesssim T$.

The predictions of the HRG model for quark number susceptibilities are compared to the lattice QCD results in Fig. (2). They compare quite well for the strangeness and baryon susceptibilities up to $T \simeq 150 \mathrm{MeV}$. As for the electric charge susceptibility, the agreement is clearly less good. Part of the discrepancy is thought to be a lattice artifact related to the mass splittings of the pseudoscalar bosons in the staggered lattice action. Finer lattice spacings and high statistics are needed to test the HRG model at low temperatures.

\section{Topology of the QCD phase diagram}

Returning to Fig. (1), one central question in the field is to establish whether or not the QCD transition becomes a sharp phase transition at some finite value of $\mu_{B}$. Due to the sign problem encountered at finite baryon chemical potential, this question is difficult to answer using lattice QCD methods. The commonly used methods are reweighting ([14], see [15] for a discussion of the method), Taylor expansion [16, 17] and analytic continuation from imaginary chemical potential (see [7] for a review). The basic idea behind the Taylor expansion method is that if the Taylor coefficients of the free energy all have the same sign, then the radius of convergence is on the real $\mu_{B}$ axis and can be determined from the asymptotic ratio of two successive coefficients. Since only the first few terms can be calculated in practice (at most $\mu_{B}^{8}$ ), it is hardly possible to prove the existence of a singularity of the partition function in this way. The question of how many terms are necessary to locate the critical point has been investigated in toy models [18, 19], where simulations at the critical point are possible and the results can be checked.

One question that can be addressed reliably in lattice QCD is whether the transition initially becomes narrower or broader as one moves away from the vertical axis. 

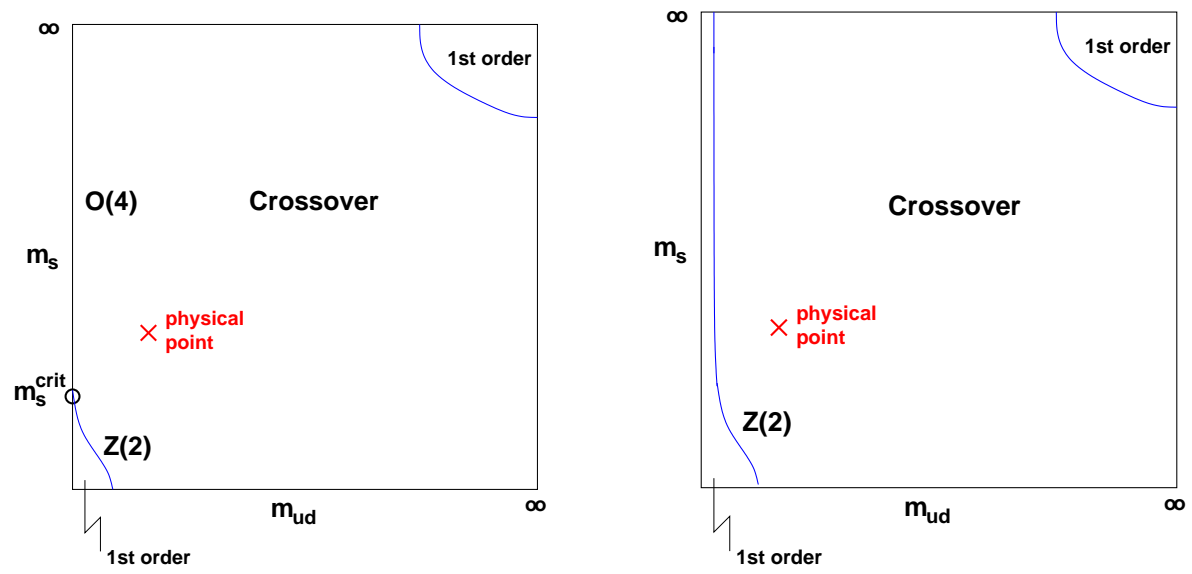

Figure 3: Two scenarios for the quark mass dependence of the order of the QCD phase transition at $\mu_{B}=0$.

\subsection{The QCD transition at small $\mu_{B}$}

The behavior of the pseudocritical line in the $\left(\mu_{B}, T\right)$ plane can be probed on the basis of the following considerations [ [ 6 ]. One chooses a physical observable $\phi\left(T, \mu_{B}\right)$ (for instance, the chiral condensate) and considers the differential in terms of the $\left(T, \mu_{B}^{2}\right)$ variables,

$$
\mathrm{d} \phi=\frac{\partial \phi}{\partial T} \mathrm{~d} T+\frac{\partial \phi}{\partial \mu_{B}^{2}} \mathrm{~d} \mu_{B}^{2}
$$

If the crossover curve is defined by $\phi$ taking a certain specific value (one could think of other criteria), then the change of the pseudocritical temperature is given by

$$
\left.\frac{\mathrm{d} T_{c}}{\mathrm{~d} \mu_{B}^{2}}\right|_{\mu_{B}=0}=R\left(T=T_{c}\right), \quad R(T) \equiv-\frac{\partial \phi / \partial \mu_{B}^{2}}{\partial \phi / \partial T}\left(T, \mu_{B}=0\right) .
$$

To probe the $\mu_{B}$-dependence of the width of the crossover, defined by $W=\left.\frac{1}{\phi} \frac{\partial \phi}{\partial T}\right|_{T=T_{C}}$, Endrodi et al. use the relation

$$
\frac{T_{c}^{2}}{W} \frac{\partial W}{\partial \mu_{B}^{2}}=T_{c}^{2}\left(\frac{\partial R}{\partial T}\right)\left(T=T_{c}\right)
$$

In the case where $\phi$ is the chiral condensate, they find for this quantity $0.030(18)$. This result suggests a very slight broadening of the transition. The main point is that the chemical potential has initially little effect on either the transition temperature or the width of the crossover.

\subsection{Dependence of the phase diagram on the quark masses}

The order of the QCD phase transition as a function of the $u, d$ quark mass and the strange quark mass is summarized in the 'Columbia plot' displayed in Fig. (3). In the 'standard' scenario (on the left), there is a tricritical point in the strange quark mass. The chiral transition in two-flavor QCD (i.e. when $m_{s} \rightarrow \infty$ ) is second order and in the $\mathrm{O}(4)$ universality class. An alternative scenario is that there is no critical point in $m_{s}$, instead the second-order transition line (in the $\mathrm{Z}(2)$ class) extends all the way to $m_{s}=\infty$. The chiral transition in $N_{\mathrm{f}}=2 \mathrm{QCD}$ is then of first order. Which of these scenarios is realized is under active investigation [20, 21, 22, 23]. See [24] and Refs. therein for the potential role of the effective restoration of the $U(1)_{A}$ symmetry around $T_{C}$. 


\section{Conclusion and outlook}

It is by now well-established that the QCD transition is a crossover. The chiral transition takes place somewhat before the partonic degrees of freedom are fully deconfined. Calculations of equilibrium properties at $\mu_{B}=0$ are at quite an advanced stage and the systematic errors are being reduced. An achievable goal in the near future is to compute several Taylor coefficients of the free energy expanded in powers of $\mu_{B}$ with controlled uncertainties. Computational methods to investigate the phase diagram at larger baryon chemical potential [15] as well as reliable methods to extract dynamical properties [25, 26] (spectral functions and in particular transport properties) need to be further developed.

\section{Acknowledgments}

I thank my collaborators Bastian Brandt, Anthony Francis and Hartmut Wittig for discussions. This work was supported by the Center for Computational Sciences in Mainz.

\section{References}

[1] J. Bjorken, Highly Relativistic Nucleus-Nucleus Collisions: The Central Rapidity Region, Phys.Rev. D27 (1983) 140-151.

[2] Y. Aoki, G. Endrodi, Z. Fodor, S. Katz, and K. Szabo, The Order of the quantum chromodynamics transition predicted by the standard model of particle physics, Nature 443 (2006) 675-678, [hep-lat/0611014].

[3] Wuppertal-Budapest Collaboration, S. Borsanyi et. al., Is there still any Tc mystery in lattice QCD? Results with physical masses in the continuum limit III, JHEP 09 (2010) 073, [arXiv:1005.3508].

[4] A. Bazavov, T. Bhattacharya, M. Cheng, C. DeTar, H. Ding, et. al., The chiral and deconfinement aspects of the QCD transition, Phys.Rev. D85 (2012) 054503, arXiv:1111.1710.

[5] C. DeTar and U. M. Heller, QCD Thermodynamics from the Lattice, Eur. Phys. J. A41 (2009) 405-437, [arXiv:0905.2949.

[6] G. Endrodi, Z. Fodor, S. Katz, and K. Szabo, The QCD phase diagram at nonzero quark density, JHEP 1104 (2011) 001, arXiv:1102.1356.

[7] O. Philipsen, Status of the QCD phase diagram from lattice calculations, arXiv:1111.5370.

[8] S. Borsanyi, Z. Fodor, S. D. Katz, S. Krieg, C. Ratti, et. al., Fluctuations of conserved charges at finite temperature from lattice QCD, JHEP 1201 (2012) 138, arXiv:1112.4416].

[9] HotQCD Collaboration Collaboration, A. Bazavov et. al., Fluctuations and Correlations of net baryon number, electric charge, and strangeness: A comparison of lattice QCD results with the hadron resonance gas model, arXiv:1203.0784.

[10] WHOT-QCD Collaboration Collaboration, T. Umeda et. al., Equation of state in 2+1 flavor QCD with improved Wilson quarks by the fixed scale approach, arXiv:1202.4719.

[11] M. Cheng, N. H. Christ, M. Li, R. D. Mawhinney, D. Renfrew, et. al., The finite temperature QCD using $2+1$ flavors of domain wall fermions at $N(t)=8$, Phys.Rev. D81 (2010) 054510, [arXiv:0911.3450]. 
[12] R. F. Dashen and R. Rajaraman, Narrow Resonances in Statistical Mechanics, Phys. Rev. D10 (1974) 694.

[13] R. F. Dashen and R. Rajaraman, Effective Elementarity of Resonances and Bound States in Statistical Mechanics, Phys. Rev. D10 (1974) 708.

[14] Z. Fodor and S. Katz, Lattice determination of the critical point of QCD at finite T and mu, JHEP 0203 (2002) 014, hep-lat/0106002].

[15] P. de Forcrand, Simulating QCD at finite density, PoS LAT2009 (2009) 010, [arXiv:1005.0539.

[16] R. Gavai and S. Gupta, QCD at finite chemical potential with six time slices, Phys.Rev. D78 (2008) 114503, [arXiv:0806.2233].

[17] RBC-Bielefeld Collaboration, HotQCD Collaboration Collaboration, C. Schmidt, $Q C D$ thermodynamics at zero and non-zero density, Nucl.Phys. A820 (2009) 41C-48C, arXiv:0810.4024].

[18] M. A. York and G. D. Moore, Exploring the Phase Diagram with Taylor Series: Epic Voyage or Just Another Bad Trip, arXiv:1106.2535.

[19] F. Karsch, B.-J. Schaefer, M. Wagner, and J. Wambach, Towards finite density QCD with Taylor expansions, Phys.Lett. B698 (2011) 256-264, arXiv: 1009.5211].

[20] B. B. Brandt, O. Philipsen, H. Wittig, and L. Zeidlewicz, Towards the Nf=2 deconfinement transition temperature with $O($ a) improved Wilson fermions: An update, AIP Conf.Proc. 1343 (2011) 516-518, arXiv:1011.6172].

[21] S. Ejiri et. al., On the magnetic equation of state in (2+1)-flavor QCD, Phys. Rev. D80 (2009) 094505, [arXiv:0909.5122].

[22] F. Burger, E.-M. Ilgenfritz, M. Kirchner, M. Lombardo, M. Muller-Preussker, et. al., The thermal QCD transition with two flavours of twisted mass fermions, arXiv:1102.4530.

[23] V. Bornyakov, R. Horsley, S. Morozov, Y. Nakamura, M. Polikarpov, et. al., Probing the finite temperature phase transition with $N f=2$ nonperturbatively improved Wilson fermions, Phys.Rev. $\mathbf{D 8 2}$ (2010) 014504, [arXiv:0910.2392].

[24] F. Basile, A. Pelissetto, and E. Vicari, Finite-temperature chiral transition in QCD with quarks in the fundamental and adjoint representation, PoS LAT2005 (2006) 199, hep-lat/0509018].

[25] H. B. Meyer, Transport Properties of the Quark-Gluon Plasma: A Lattice QCD Perspective, Eur.Phys.J. A47 (2011) 86, [arXiv:1104.3708].

[26] H. B. Meyer, Lattice QCD and the Timelike Pion Form Factor, Phys.Rev.Lett. 107 (2011) 072002 , [arXiv:1105.1892]. 